\title{
Marcel Mauss (1872-1950)
}

Sociologue et anthropologue français

\author{
(1938) \\ "Fait social \\ et formation du \\ caractère"
}
Un document produit en version numérique par Jean-Marie Tremblay, bénévole, professeur de sociologie au Cégep de Chicoutimi
Courriel: jean-marie tremblay@uqac.ca
Site web pédagogique : http://www.uqac.ca/jmt-sociologue/

Dans le cadre de la collection: "Les classiques des sciences sociales"

Site web: http://www.uqac.ca/Classiques_des_sciences_sociales/

Une collection développée en collaboration avec la Bibliothèque

Paul-Émile-Boulet de l'Université du Québec à Chicoutimi

Site web: http://bibliotheque.uqac.ca/ 
Cette édition électronique a été réalisée par Jean-Marie Tremblay, bénévole, professeur de sociologie au Cégep de Chicoutimi à partir de :

\section{Marcel Mauss,} [sociologue et anthropologue français, 1872-1950]

\section{"Fait social et formation du caractère"}

Un article publié dans la revue Sociologie et sociétés, vol. 36, no 2, automne 2004, pp. 135-140. Montréal : Les Presses de l'Université de Montréal.

Polices de caractères utilisée :

Pour le texte: Times New Roman, 14 points.

Pour les citations : Times New Roman 12 points.

Pour les notes de bas de page : Times New Roman, 10 points.

Édition électronique réalisée avec le traitement de textes Microsoft Word 2004 pour Macintosh.

Mise en page sur papier format : LETTRE (US letter), 8.5 " x 11"')

Édition complétée le 31 juillet 2005 à Chicoutimi, Ville de Saguenay, province de Québec, Canada.

\section{Fait avec}




\section{Marcel Mauss (1872-1950)}

Sociologue et anthropologue français

(1938)

"Fait social

et formation du caractère"

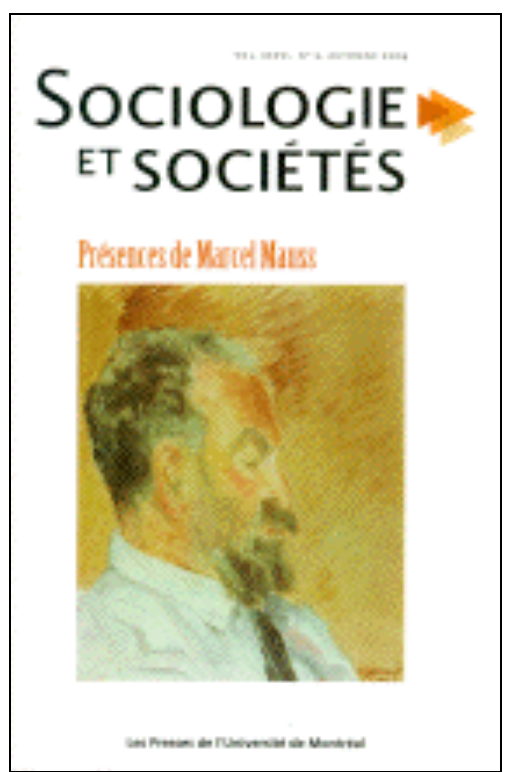

Un article publié dans la revue Sociologie et sociétés, vol. 36, no 2, automne 2004, pp. 135-140. Montréal : Les Presses de l'Université de Montréal. 


\title{
Présentation du texte
}

\author{
Par Marcel Fournier, \\ Sociologue, Université de Montréal, \\ Directeur de la revue Sociologie et sociétés.
}

Automne 2004.

CE TEXTE INÉDIT de Marcel Mauss a été découvert au moment où, dans le cadre de la recherche qui devait aboutir à la publication de notre livre sur Marcel Mauss, nous effectuons l'inventaire du Fonds Hubert-Mauss au Collège de France. Il s'agit de notes préparatoires pour une communication qu'il devait présenter au Congrès international des sciences anthropologiques et Ethnologiques qui se tint à Copenhague dans le courant de l'été $1938{ }^{1}$.

Ces notes se présentent sous la forme d'un manuscrit de neuf feuillets, écrits d'un seul côté. Très difficile à déchiffrer, ce brouillon, hâtivement jeté sur le papier, est manifestement incomplet, s'achevant notamment sur une série d'indications de style télégraphique, sans doute destinées à être utilisées en guise d'aide-mémoire.

À l'occasion de ce congrès, Mauss s'est rendu au Danemark en compagnie de huit de ses élèves : Marcel Griaule, Anatole Lewitszki, Georges-Henri Rivière, Thérèse Rivière, André Schaeffner, Jacques Soustelle, André Varagnac et Paul-Émile Victor. Mauss s'y montre fort actif, car en plus de présenter sa communication, «Fait social et

Des fragments de ce texte ont été publiés dans l'Ethnographie, 93, no 1-2, 1997, pp. 9-14. 
formation du caractère », il participe aussi à un débat sur «les relations entre les aspects religieux et sociologiques du rituel ». Dans les Actes du Congrès international des sciences anthropologiques et ethnologiques (Copenhague, Einar Munksgaard, 1939, P. 199), se trouve un compte rendu succinct de la session à laquelle participe Mauss.

Marcel Mauss connaît-comme disent gentiment ses amis-son «moment de gloire» (lettre de Paul Fauconnet à Marcel Mauss, 8 août 1938). Fondateur de l'Institut d'Ethnologie de Paris en 1925, professeur au Collège de France, où il occupe la chaire de sociologie, Mauss vient, en mai 1938, d'être élevé à la présidence de la section des Sciences religieuses. Bref, on le considère alors comme le " protecteur fidèle de toute recherche ethnologique en France » (Carl Sachs: Les instruments de musique de Madagascar, Paris, travaux et mémoires de l'Institut d'Ethnologie, tome XXVIII, 1938, p. viii).

$\mathrm{Au}$ moment où Mauss se rend à Copenhague, il a en préparation une autre communication, à laquelle il fait référence, sur la notion de personne. Cette communication, intitulée «Une catégorie de l'esprit humain : la notion de personne », sera présentée à l'automne de cette même année devant les auditeurs de la Huxley Memorial Lecture, avant d'être publiée dans le Journal of the Royal Anthropological Institute. " Fait social et formation du caractère » et "Une catégorie de l'esprit humain » constituent les deux dernières grandes prestations scientifiques de Marcel Mauss avant le début de la Seconde Guerre mondiale.

Marcel Fournier 
Je remercie le président, Monsieur le Professeur Rubin, dont nous connaissons le nom en France et les travaux de psychologie, dont je sais la compétence en matière précisément d'ethnologie psychologique, de science du caractère $\mathrm{du}$ point (de vue) psychologique, d'avoir bien voulu me demander d'ouvrir la discussion en apportant un autre point de vue que le sien, également valable, celui du sociologue que je suis. D'ailleurs, le sujet des débats de cette section, à laquelle j'adhère avec pleine conscience, est précisément : Civilisation et Caractère. Et vous me permettrez de considérer pendant toute cette dernière heure le terme «civilisation » comme équivalant au terme « société ».

$\mathrm{Au}$ surplus, peut-être savez-vous que la sociologie, objets, technique, dont je suis l'ouvrier, West nullement pour moi, n'était nullement pour Durkheim, mon maître et mon oncle, exclusive de toute considération psychologique des mêmes faits, dans l'individu et dans son milieu social à la fois. Et peut-être l'honneur que vous me faites est-il aussi dû à mes efforts pour traiter de façon concrète et double des rapports réels, dans l'homme total, seul sujet de [illisible] et des «histoires» de sociétés où nous puisons nos faits -donc psychologique et social. Peut-être même un certain nombre d'entre vous connaissent mes travaux sur l'expression obligatoire des sentiments, les effets suggestifs et physiques de l'idée de la mort, sur les techniques du corps-prix que j'ai, peut-être un peu malgré moi, payé à la Société de Psychologie française, pour l'honneur de m'avoir pendant plusieurs années maintenu comme son président.

Mais trêve de ces considérations à la fois générales et peut-être trop personnelles. Vous trouverez d'ailleurs peut-être que ce qui suit da pas grande importance, et ne vaut pas la peine de tant de rites et cérémonies, formalités et circonlocutions. Mon excuse est l'extrême généralité du problème que vous avez posé. Mon effort va plutôt être de le restreindre et de le clarifier. Peut-être un savant d'École française manque-t-il de vues du complexe, du confus, de l'au-delà de ce qu'il 
inclut dans sa démonstration. Mais, pour nous, il s'agit d'apercevoir sinon le tout des faits, au moins celui de quelques faits et de les expliquer, de les décrire en termes vrais, c'est-à-dire clairs, distincts et sûrement adéquats (comme disait Spinoza).

Appelons si vous le voulez bien «faits de civilisations 》 tous les faits sociaux. C'est une évidence : une société étant l'ensemble des hommes, des choses qu'ils possèdent, et des représentations de ces hommes et des pratiques qu'ils suivent: techniques, arts, religion, droit, etc. L'ensemble de ces représentations collectives et de ces pratiques forment sa mentalité collective. Celle-ci se caractérise à son tour, tout comme la mentalité d'un individu, par les [proportions] et la nature des divers comportements dont elle est capable, par ce qu'on appelle, d'un assez mauvais mot, civilisation, et d'un plus mauvais mot, kultur, culture, et que nous appellerons son éthologie, son caractère.

La définition que je vais vous proposer du caractère individuel est exactement de même sorte. Sans aucunement verser dans un substantialisme psychologique, nous pouvons cependant dire qu'il existe des individus, des personnes : chaque individualité -humaine tout au moins -et sûrement à de moindres degrés tout animal supérieur a son comportement général à lui : un tout de facultés, d'habitudes, de façons de percevoir et de réagir, d'humeurs, de forces physiques et mentales, singulier, non pas simplement parce que l'individu se distingue par la position de son corps, mais aussi parce que la série d'évènements dont à est le siège est vraiment un tout dans le temps et l'espace. Nous disons un tout, un tout organique, et non pas un simple total. Car quoique Marcel Mauss ne soit pas l'intégration, la somme des événements psychologiques et physiologiques, qui passe à tel endroit et passera dans tel et tel temps, cette intégration est autre chose, bien plus qu'une somme algébrique d'événements ou même que la limite de leur courbe. Or, s'il est un succès de la psychologie récente -auquel le Dr Rubin n'est pas étranger, auquel a contribué toute l'école des tests (Binet, Simon et les autres) et qui a abouti aux formules qu'a laissées Spearman - c'est bien ce bertillonnage individuel, cette anthropométrie de la mentalité même. Et de même que le bertillonnage somatologique arrive à identifier un individu l'observation des empreintes digitales est probablement 
prodigieusement ancienne -, de même on arrivera à formuler le caractère de Marcel Mauss, de ce cobaye que j'essaie d'être en ce moment devant vous. C'est ce que savent bien les romanciers tout court, et les romanciers d'analyse en particulier, comme fut notre Proust.

Les définitions nous mettent sur la voie. Voyons où, quand et comment cette mentalité collective s'impose et s'implante dans l'individu, et comment l'individu se construit d'éléments sociaux et d'éléments individuels de son histoire biologique et sociale. Enfin, comment aussi à peut agir sur la société comme celle-ci agit sur lui.

Nous prendrons quelques cas concrets de ces rapports entre individu, espace et société, dans toutes sortes de sociétés - une certaine quantité, dites primitives - mais aussi d'autres, et même des cas très proches de nous. Nous les rangeons en désordre, sans distinction d'origine géographique ou historique, mêlant le social et l'individuel, faisant défiler des Australiens et des Cheyennes avec des japonais ou des Français. Le temps nous manque de présenter mieux nos échantillons.

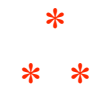

Le point où On peut le mieux apercevoir cette action est dans l'éducation et même dans le dressage de l'enfant. L'homme est un animal qui éduque et dresse ses enfants, et les dote consciemment, et depuis quelques milliers d'années en constant progrès dans les pays dits civilisés. De même [mots illisibles] ses enfants. Non seulement par nécessité biologique mais parce que la vie des adultes [conforme] les moyens de [vie] même instinctifs des jeunes enfants, par exemple les moyens de transport de l'enfant, ou de propreté. Je ne parle même pas des tortures du maillet qui furent infligées à ma génération, je parle de l'échafaudage de soins qui permet par exemple de dresser et d'éduquer l'enfant, très tôt, vers la réglementation de ses besoins. Un exemple : un couple d'instituteurs distingués, d'ailleurs prédestiné - il est devenu un des meilleurs administrateurs d'hospice d'aliénés- a su habituer ses deux enfants (qui sont devenus de distingués psychiatres 
et neurologistes) à des heures précises d'urination [ill.]. Je n'ai pas besoin de rappeler le rappel des heures de repas sans [ill.] appétit. L'enfant sait très vite, tout endormi qu'il paraisse. D'autres exemples sont non moins connus. Par exemple, les psychanalystes de la libido [savent] fort bien que la façon dont l'enfant est longtemps porté par la mère, souvent directement sur sa peau, plus ou moins bien tenu, donne à l'enfant des habitudes et des sensations inoubliables. je me garderai d'insister là-dessus, ainsi que cette très précoce - peut-être tout de suite après la première semaine - éducation du cri de l'enfant. Dans ma famille, Durkheim, pour ses enfants, à l'indignation de ses sœurs, a [ill.] laisse pleurer ses enfants sans les « prendre dans ses bras ». Faute d'éducation [rature]. Sur ce point comme bien d'autres gymnastiques nudisme, etc.-, vous serez [ill.] par moi [ill.] est la part que les pédiatres scandinaves ont prise et prennent encore au progrès de la pédiatrie en tout point.

J'ai exprès indiqué comment ces problèmes se posent dans nos sociétés à nous. Dans celles qui relèvent de l'ethnographie - ne croyez pas que je crois en ces divisions -, l'éducation se poursuit quelquefois, comme chez nous encore celle du savant (peut-être pas toujours du professeur). Le magicien est aussi curieux de rites que nous de faits. D'ailleurs, même chez nous, les poètes sont restés d'éternels [ill.] [phrase ill.]. La vie, pour un noble Kwakiutl ou pour un [ill.] polynésien, est une sorte de perpétuelle édification de la science et du caractère de l'homme (nous disons encore " édifier », et soi-même par sa piété et les autres par l'exemple). Dans le cas du nord-ouest Américain, le jeune homme, puis l'adulte - et ceci ne cesse qu'avec la véritable retraite-passent leur vie à de perpétuelles initiations, avec extase et possession obligatoires, nécessaires pour obtenir les titres, prérogatives, mariages, biens, rangs politiques sociaux, prestige. La fin de cette existence est l'entrée dans la société des retraités chez les Kwakiutl [ill.]. Et le plus beau fait que je connaisse [phrase ill.].

En général, tous les Indiens de la côte nord-ouest ont des institutions du même genre, et une partie des Indiens du centre et de l'est en ont d'autres de même fonction sinon de même nature. Et nous nous trouvons tout de suite en présence d'un immense problème, fort contesté, celui du manitouisme et de la recherche de l'esprit personnel. 
Si vous le voulez bien, je vous ferai entièrement grâce des contestations qui se sont élevées à son sujet. Le manitou [révèle] est-il le produit de l'autorité religieuse ou est-il la source de la religion ? Les principes ne me concernent pas, pas plus que le biologiste da à s'imaginer si c'est la poule ou si c'est l'œuf qui fut l'être premier. Je n'ai qu'à vous parler des rapports. Et je me contenterai du dire de cette grand-mère Winnebago à Radin, à propos de son petit-fils qui allait avoir ses révélations : "je lui dis quels sont les êtres qu'il peut prétendre voir se révéler à lui. » On peut vérifier ce même fait dans l'importante auto(bio)graphie de Crashing Thunder rédigée par [Winuly ? ${ }^{2}$ ] et arrangée par le même Radin ${ }^{3}$. Je n'en finirais plus de vous citer des faits de ce genre. Mais il nous faut encore voir les effets de cette autosuggestion préfigurée par le droit à être hanté de tel ou tel esprit, soit un de ces hommes qui fut tel et tel esprit, de tel et tel animal, de tel et tel fantôme, qui réincarna tel ou tel ancêtre pendant telle et telle transe répéta -ici et là à chaque grande fête, ce même personnage- ou encore plus, qui en répéta un autre au titre de telle fonction, de tel héritage, de tel événement-puis encore un autre qui a été-c'est le cas de tout le shamanisme sibérien, nord-américain et russe. Nos amis scandinaves savent ces choses mieux que moi-l'hôte successif d'un nombre d'êtres qui lui sont familiers, pour lesquels, par sa transe et sa crise, il peut se [ill.] et vivre. Sa vie est faite fondamentalement de son caractère. Voyez l'admirable petite statue que Mme [Thallitzea ?] a faite de [ill.] d'[Amassalik]. Elle exprime merveilleusement les traits profonds dont le rôle magique et religieux de [ill.] son expérience [ill.] de ce rôle marque tout l'habitus de l'homme.

Mais ces faits sont trop évidents et trop particuliers, trop [exagérés] pour emporter peut-être votre adhésion. Cette prise de possession de l'individu par son rôle est également le fait de nos civilisations et de nos mentalités à la fois collectives et individuelles. Tout notre théâtre et toute notre littérature en est (sic) pleine. Au [ill.] l'un des plus rares mérites, datant peut-être de la Renaissance et [venant] de quelques minorités du nord et du sud de l'Europe, a été d'édifier [l'individu] à

S'il faut lire «Hensley », ce serait une erreur de Marcel Mauss : il s'agit en réalité de Sam Blowsnake.

3 Paul Radin a publié en 1926 Crashing Thunder, une version retravaillée de The Autobiography of a Winnebago Indian (1920). 
être lui-même plus que son personnage. C'est notre Montaigne qui disait de lui et de son président du Parlement de Bordeaux, président au « Marteau » (le grand [ill.] qu'il était le seul, lui « Montaigne », il n'avoir pas [Martel] en lui. [ill.]. C'est par opposition à la vie collective que l'individu -l'être-, de pure conscience et liberté, s'est créé dans la vie. Ce que nous enseignent la sociologie et plus généralement l'histoire, c'est combien est récente la [ill.] de la notion de personne. Dans une conférence ${ }^{4}$ que je dois à une autre honorable institution, j'ai promis de décrire ce que je crois être l'histoire de la notion de personne humaine. Ici je [n'espère] vous dire qu'une chose, c'est qu'elle n'est entrée dans la philosophie, suffisamment complète, qu'au début du dernier siècle, à la fin du [ill.], de Swedenborg et de Kant.

L'homme a été longtemps un personnage avant d'être une personne. Sa place, son rang, son rôle ont été son tout avant d'être sa simple vie intérieure. Il s'agit par le temps qui court de fabriquer des « personnes humaines ». Nous le pouvons enfin.

Je voudrais maintenant vous parler non plus des rapports de cette formation sociale du personnage (Epictète disait -parlant son Grec latinisé- : « Sculpte ton masque, ton... », à Marc-Aurèle pour lui dire : «Sois ton idéal à toi. ») mais de la formation claire de l'individu et de sa fonction-non pas dans des sociétés [ill.] ou même relativement primitives-mais dans celles-ci. La conscience claire que l'individu a de soi et des autres est un «trait de civilisation » de nos sociétés à nous (j'applique, vous le voyez, la méthode de l'ethnographie la plus historique à notre histoire à nous). £individu est enfin devenu le sujet et l'objet, l'agent responsable de la vie sociale. Ce qu'il était inconsciemment, prisonnier de son rang et de ses habitudes, il l'est devenu consciemment. Il se sait son pouvoir. Je ne veux pas vous ennuyer de ma confession de guerre. Dans l'immense Machine, l'horrible combat moderne, ce ne sont plus les princes ou les [ill.] qui sont les héros. C'est le «soldat inconnu» qui «tient» pour ses

Marcel Mauss, «Une catégorie de l'esprit humain : la notion de personne, celle du "moi", un plan de travail » (Huxley Memorial Lecture, 1938), Journal of the Royal Anthropological Institute, 68,1938, pp. 263-281, in Marcel Mauss, Sociologie et anthropologie, Paris, PUF, 1950 ; 4e édition augmentée, 1968, pp. 331-362. 
camarades et pour lui, c'est [ill.] qui [ill.]. Les armées de la dernière guerre étaient des armées de citoyens.

C'est maintenant l'individu qui est à la source du changement social. Il l'a toujours été, mais il ne le savait pas. Les lois venaient de ses princes et de ses religions. Ses coutumes sortaient de ses techniques. Celles-là, enfoncées dans l'artisanat, lui laissaient ingéniosité plus qu'innovation devant lui. L'ingénieur est venu lentement depuis les premières machines. Les sociétés modernes changent plus vite que jamais (Social Trends). Il s'agit, sous peines cruelles, [quatre mots illisibles] de ce rôle de l'individu.

Non pas qu'il faudrait tomber dans les folies éducatives du « Culte des héros ». Le [ill.] romantique est moins beau que l'histoire de tel ou tel savant, de tel ou tel vrai penseur, inventeur ou ministre. Il s'agit de donner aux hommes le sens de ce qu'ils sont par la société, le sens des changements sociaux dans la société qu'ils doivent conserver pieusement et (vouloir) fortement.

Vous posez ici le problème des changements; permettez-moi, en conclusion, de vous donner des indications sur ce point.

[Suivent, pour conclure, quelques indications elliptiques, impossibles à décrypter.]

Fin du texte 